\title{
A COMPARATIVE RETROSPECTIVE STUDY OF POISONING CASES IN CENTRAL, ZONAL AND DISTRICT HOSPITALS
}

\author{
${ }^{1}$ Deepak Pokhrel*, ${ }^{2}$ Sirjana Pant, ${ }^{2}$ Anupama Pradhan, ${ }^{2}$ Saffar Mansoor \\ ${ }^{1}$ Currently working as lecturer in Department of Pharmacy, Asian College for Advance \\ Studies, Jawalakhel, Lalitpur, Nepal \\ ${ }^{2}$ Department of Pharmacy, Kathmandu University, GPO Box: 6250, Kathmandu, Nepal \\ *Corresponding author: todipak@gmail.com
}

Received 28 August; Revised 18 September

\begin{abstract}
The 582 data collected from Central, Zonal and District level Hospitals, which occurred from Magh 2057 to Poush 2058 produced significant results. Various parameters (age, sex, marital status, time of ingestion, month of occurrence, Agent responsible for the incidence, type of poisoning, outcome and duration of treatment) were analyzed. Similarly the pattern of frequency distribution of the above-mentioned parameter in central, zonal and district level of hospitals were compared.
\end{abstract}

Hypothesis were made and tested. Females were found to be more susceptible to the intentional incidence than male $(\mathrm{p}=0.000)$. Intentional poisoning for unmarried male was found to be more (34\%) than for female. On the contrary, intentional poisoning in female was high in case of married subjects $(57 \%)(\mathrm{p}=0.000)$. The most common type of poisoning causing the intentional attempt was organophosphates $(40 \%)$ followed by Phosphides (14\%). On the other hand unintentional poisoning was most common due to the unclassified and other agents $(8 \%)$, which were followed by hydrocarbons $(6.3 \%)$. (p $=0.000)$. The most common organophosphate used for intentional attempt for poisoning is methyl parathion (31\%) when compared with other groups of organophosphates $(26 \%)$. Sedative and hypnotics were the most common type of drugs (43\%) used for intentional attempt. Nighttime incidence was more frequent (31\%). Homicidal poisoning occurring in both central and district hospital were equal being 3.6\% of the total types of attempt of incidence. Most of the cases as obvious were found to be cured (84.8\% out of total cases). The zonal hospital shows the highest cure rate $(89.2 \%)$ followed by district hospital (74.2\%)-the comparison however excludes central level hospitals. The mortality however is similar in both hospitals ( $4.4 \%$ and $4.5 \%$ respectively in zonal and district hospitals).

Accidental intake of hydrocarbon was found to be most frequent in the children (33\%) while intentional intakes of other agents were more frequent in adults (97\%). The rainy season was the most common season for the attempt of intentional incidence $(47.8 \%)$ adding to mortality. The most common age group of subjects exposed to intentional attempt that adds to the mortality is $15-24$ years $(39.1 \%)$. The TYPE, primarily adding to mortality was intentional intake $(91.3 \%)(\mathrm{p}=0.008)$. Most of the intentional incidence adding to mortality is caused by organophosphates $(66.7 \%)$ followed by Phosphides (19\%). Subjects between age group 15-24 are more likely to cause the intentional exposure $(44 \%)$ while children below five years are more likely to cause accidental 
KATHMANDU UNIVERSITY JOURNAL OF SCIENCE, ENGINEERING AND TECHNOLOGY

VOL. I, No. V, SEPTEMBER 2008, pp 40-48.

exposure $(6 \%)(\mathrm{p}=0.000)$. Seasonal comparison showed a high incidence in rainy season (24\%) as compared to that of autumn $(23 \%)$.

Key words: Central Hospital, Zonal Hospital, District Hospital, Aluminium Phosphide, Intentional Poisoning.

\section{INTRODUCTION}

Poison is a substance capable of producing damage or dysfunction in the body by its chemical activity. It can enter the body in various ways to produce general or local effects (limited to the eyes, skin, lungs, etc.). All cases of poisoning that result from accidental use of drugs and chemical substances, or the use of drugs by children due to curiosity, are known as accidental or non-intentional poisoning. Poisoning is a qualitative term used to define the potential of a chemical substance in acting adversely or deleteriously on the body.

Advances in technology and social development have resulted in the availability of most drugs and chemical substances in the community. These chemical substances pose a significant threat due to their poisonous effect the extensive use in medicine, agriculture, industry and residential environments.

Pesticides and Drugs are the common agents causing the incidence. Whether intentional or accidental, it is the easy access to these substances that significantly adds to the incidence. Narcotic Drug Control Act, 2038, prohibits misuse of Narcotics and Pesticide Act 2048 has similarly prohibited the misuse of pesticides. However improper implementation of these has lead to such problems. Pesticides like methyl parathion (metacid) are very commonly used for intentional poisoning. Pesticides like aluminium phosphide, having mortality rate more than $50 \%$ are easily available in the market

In suicidal cases the root cause is other than the poison itself. It is the psychological state of the patient and treatment of the poisoning is not the sole treatment, a psychiatric counseling is a must. Suicidal cases can be divided into suicide and attempted suicide or as true suicide attempts in which the goal is to die and suicidal gesture in which there is some other goal such as to "cry for help". Whereas in the case of accidental poisoning, being a bit more careful can solve the problem. Children and old age are usually prone to accidental poisoning, so the guardians must be suggested of the preventive measures. Poisoning due to drugs and chemicals is influenced to a large extent by the population's socioeconomic and cultural status.

Poisoning either accidental or intentional is a matter of major consideration in relation to the health care systems. The incidences are increasing day by day. A study showed an increase from 3.2\% (BS 2045-2048) of the total admission to 4.2\% in year BS 2052-2055 in Patan Hospital, Nepal (Vaidya and Shrestha, 1999). Another report shows that incidence of acute poisoning increased by $19.23 \%$ in one year $1997-1998$ and by $3.86 \%$ from Jan 1999 - Jun 2001 (Bharati et al. 2001). This also indicates the increasing incidence of poisoning. A steady increase in the number of percentage of poisoning cases admitted in Tribhuwan University Teaching Hospital (TUTH), increased from $0.1 \%$ to 0.5\% during year 1986-1992 (Kafle and Gyawali, 1992). Similar results were observed in the studies in Bir Hospital around year1981-1982 to 1984-1985 (Pokhrel and Gurung 
1987). These trends are common not only in Nepal but also in developed countries like USA and other European countries. During year 1990-1995 mortality rate due to poisoning showed a $25 \%$ raise (Sobhani et al. 1995).

These studies indicate a growing need of attention towards poisoning. These cases are mainly of intentional or suicidal rather than accidental one (Sobhani et al. 1995; Kafle et al 1989; Lohani 1995). But another study showed the accidental cause of poisoning to be more frequent than intentional one (Bharati, 2001). This trend can be important in pointing the similar results in study from USA and other developed countries (Sobhani et al. 1995). The studies showed that the intentional poisoning are due to various causes and the major of which was the family conflict followed by accidental intake (Kafle and Gyawali 1992) while other researchers emphasizes on the cause due to mental illness (Berky 2000). The same research work also indicate male to be more prone to suicidal attempts, which is in contrast to the results obtained from the Nepalese workers (Vaidya 1999; Bharati 2001; Sobhani 1995; Pokhrel 1987; Kafle 1989) Still other work from Zimbabwe showed unemployment to be major cause (Hobun and Nahachi 1996).

The type of poisoning agent differs from country to country. In Nepal, the most common poisoning agent are organophosphates (Vaidya 1999; Bharati 2001; Kafle 1989; Kafle 1992; Lohani 1995). However, the use of organophosphate are gradually overcoming by the use of Phosphides and similar rodenticides, due to their fast and more effective action (Vaidya 1999 and Lohani 1995). The use of organophosphates(OP) were also found to be common in Iran (Sobhani et al. 1995). But, it was found that anticholinergics were the major agent for the incidence in USA (Bruns et al. 2000).

Majority of the poisoning cases are found in women (Vaidya 1999; Kafle 1989; Pokhrel 1987; Lohani 1995). These data are supported by other researchers from Iran and USA. (Sobhani 1995; Bruns 2000). However, a survey in Nepal Medical College Teaching Hospital(NMCTH) showed the majority of incidence in males $53.84 \%$ around the year 1998 Dec to 2000 June. (Bharati et al. 2001). Similar, results were observed in a work from Ethiopia which supports male being more prone to poisoning (Bekry, 2000).

The other factors to be considered in the study of Poisoning Cases are of children. Children are generally curious in nature and they try different things due to their nature. This 'trying' may cause serious accidents to them. The most common type of accidental poisoning associated with children was found to be due to hydrocarbons (Bharati 2001 and Lohani 1998) It has been found that even a small amount of hydrocarbon e.g. Kerosene 1-5 ml can produce severe or even fatal symptomatology (Lohani et al. 1998).

The use of Phosphides is increasing these days due to their high mortality rate of $59 \%$ (Lohani, 2000) and there is no specific antidote for this agent. It is also called as "Pastilla del armour" or tablets of love in Nicaragua (Sergio Salazar), due to its high mortality rate and usefulness in suicidal attempts. There are no any analytical procedure for the detection of Phosphine residues (Sergio Salazar, Nicaragua). This is because Phosphides upon contact with water releases Phosphine, which is very toxic gas (Lohani, 2000). This gas may cause pleural effusion which is responsible for $37-100 \%$ of mortality in children (Lohani 1995). It may also affect the first aiders if the necessary precautions in handling of the patient is not done.

\section{OBJECTIVES}


Many studies have been conducted in different field of health sciences in Nepal but very few studies have been done as regards poisoning cases. Growing awareness of the problems should stimulate attempts to develop policies to control the misuses. This study similarly aims to provide an outline of these cases occurring in Nepal so as to create a concern from the government and more seriousness in the implementation of the policies so made.

\section{METHODOLOGY}

\section{Study Design}

A retrospective study of poisoning cases was conducted at different level of hospitals in Nepal.

\section{Sampling}

The following six hospitals were purposely selected for the data collection.

\begin{tabular}{|l|}
\hline Two central (Bir Hospital and Tribhuwan University Teaching hospital), \\
\hline Two Zonal (Janakpur Zonal Hospital and Gandaki Zonal Hospital) and \\
\hline Two district (Bhaktapur Hospital and Siraha District Hospital) Hospitals \\
\hline
\end{tabular}

\section{Data Collection}

Documents related to poisoning cases between Magh 2057 and Poush 2058 were sorted out in each hospital. Data were collected from treatment records/copy of prescriptions using a structured format (Annex). The key information included in the structured format were about age, sex, marital status, time of ingestion of the poison, name/type poisoning agent and outcome of the treatment.

In total information about 582 cases were collected.

\begin{tabular}{|c|c|}
\hline Name of the Hospital & No. of Cases \\
\hline Bir Hospital & 155 \\
\hline Tribhuwan University Teaching hospital & 187 \\
\hline Janakpur Zonal Hospital & 41 \\
\hline Gandaki Zonal Hospital & 131 \\
\hline Bhaktapur Hospital & 43 \\
\hline Siraha District Hospital & -25 \\
\hline & 582 \\
\hline
\end{tabular}

\section{Hypothesis Setting}

The following assumptions were made considering all the six hospitals.

1. Female are more prone to intentional poisoning

2. Marriage reduces intentional poisoning in male but increases in Female

3. The commonly used agent for intentional poisoning is Organophosphates among which methyl parathion is the most common

4. Poisoning incidence increases during night time (after 8:00pm-before5:00am)

5. Intentional poisoning mostly occurs during Rainy season further adding to mortality

6. The age group prone to poisoning is $15-24$ and the type is usually intentional, further adding to mortality

7. Organophosphates is the most common agent causing mortality

8. Maximum duration of managements 2-4 days 
9. Homicidal cases are encountered mostly in the valley

10. Sedatives and Hypnotics are the most common drugs used for poisoning

11. Children are more prone to accidental poisoning due to Hydrocarbons

One assumption was made considering only the district and zonal level hospitals.

1. Cure rate is more in Zonal level Hospitals

These assumptions were tested by null hypothesis, which assumes that there is no relationship between the above-mentioned parameters in the above-mentioned ways. Statistical tests were used to find the significance of the hypothesis so made.

\section{DATA ANALYSIS \& RESULTS}

Frequency distribution was studied for the following cases with the respective results:

\section{Results of Overall Frequency Distribution.}

$\circ$ Most of the cases were encountered in central hospital (58.8\%) and least in district hospital (11.7\%)

- Subjects between the age of $14-24$ years were mostly prone to the incidence $(49 \%)$.

- Female are more common to the incidence $(51.4 \%)$

- Rainy season was the season when most of the incidence occurred (31.1\%)

○ Nighttime was the TIME when most of the incidence occurred (15.6\%)

- Married subjects are more prone to the incidence $(37.1 \%)$ than unmarried $(30.6 \%)$

$\circ$ Intentional poisoning $(56.4 \%)$ is the most TYPE of the incidence

- Cure rate was found to be $69.8 \%$ whereas mortality was found to be $4.8 \%$

- The duration of treatment was mostly less than or equal to one day $(41.1 \%)$

- Organophosphates (43.0\%) are the most common AGENT causing the incidence.

$\circ$ The average days of the treatment of the incidence was found to be 3.34 days. However most of the treatment were completed within one day (mode $=1$ day). The standard deviation showed a wide distribution of the number of days required for the treatment.

\section{Results of Regional Distribution.}

- All the three HOSPITALS providing the treatment indicates the most prone AGE GROUP of the subject to be $15-24$ years $(50.1 \%)$.

- Female were more prone to the incidence $(54 \%)$ than male $(50 \%)$ in central hospital while the opposite results were obtained in zonal hospital.

- Poisoning incidence is most frequent in rainy season (31.3\%) in all the hospitals.

- The incidence is most frequent during nighttime (30\%) in total of all hospitals.

- Married subjects were more frequent in central hospitals (38\%) as compared to that of unmarried subjects of zonal hospitals (16\%).

- Intentional poisoning was found to be highest in central hospital (60.6\%) and lowest in district hospital (4.3\%). Similarly accidental poisoning was found to be highest in zonal hospital (11.2\%) and lowest in District hospital (1.8\%).

- The highest cure rate was found to be in zonal hospital $(89 \%)$ followed by district hospital (73\%) and CENTRAL HOSPITAL (66\%). Similarly, the mortality was found to be highest in central hospital.

- In the central and zonal hospital most of the cases are treated within one day ( $26.1 \%$ and $18.5 \%$ respectively) while in district hospitals it is two days (6\%). 
Thus central and zonal hospitals seem to provide better, faster and efficient treatment.

- It was observed that the most common agent used for the incidence to be Organophosphates (43\%) followed by Phosphides (18.4\%). However in the zonal hospital it was found that Phosphides were the most common agent $(8.6 \%)$

\section{Results of Hypothesis Testing}

The analyses of the data were done with the aid of "SPSS Ver 10.0.1 " software. Pearson Chi-square test was done to test the significance of the hypothesis. Following results were obtained:

$\circ$ The sex of the subjects is dependent upon the types of the attempt of the incidence $[\mathrm{p}$ value $=0.000]$. Females $(43 \%)$ are found to be more prone to intentional incidence than male (32\%) subjects. But opposite pattern was observed for unintentional attempt where male (16\%) being more prone to the incidence than female $(9 \%)$.

- The marital status of the subjects attempting the incidence was found to play important role in the type of the attempt of incidence by subjects of different sex. Unmarried male were more prone to intentional attempt (34\%) than married male $(32 \%)$, while an opposite relationship was observed for unmarried female $(26 \%$ who were less prone to the intentional incidence than married female (57\%). Thus marriage adds to intentional attempt of incidence for female and viceversa. [p value $=0.000$ ]

○ Organophosphates (40\%) were most common agent used for intentional attempt of incidence followed by Phosphides $(15 \%)[\mathrm{p}$ value $=0.000]$. Similarly, for unintentional types (8\%) of attempt of incidence, unclassified and other types of agents are common which is then followed by hydrocarbons $(6 \%)$. The unclassified and other types of agents were prominent due to large variety of items in the variable. So, as compared individually, hydrocarbons are most frequent for unintentional attempt of the incidence.

- Among Organophosphates, methyl parathion (31\%) is most common agent responsible for the intentional attempt of the incidence [ $p$ value $=0.091]$.

- Among drugs, sedative and hypnotics (43\%) were responsible for intentional attempt of the incidence. However, the statistical significance is questionable [p value $=0.813$ ], it represents only the dependence between the two variables i.e. types of drugs responsible for incidence and the type of attempt of the incidence.

$\circ$ The time of ingestion of the agent by the subject is independent of the type of attempt of the incidence $[\mathrm{p}$ value $=0.999]$. However incidences occur more frequently during night $(31 \%)$.

- Homicidal incidences were more frequent in central and district hospitals $(3.6 \%$ each). However, this was not significant at $\mathrm{p}=0.05$ level of confidence.

- Zonal hospital has a higher cure rate (89\%) than district hospital (74\%) and mortality was also higher in district hospital (4.5\%), which differs slightly from zonal hospital $(4.4 \%)$. [p value $=0.004$ ]

○ Unintentional incidences in children are mainly caused by hydrocarbons (33\%) [ $\mathrm{p}$ value $=0.000$ ] as compared to other agents $(17 \%)$ while intentional attempts committed by adults (97\%) are more common due to other agents.

- Intentional incidence adds to mortality in rainy season (48\%) as compared to unintentional incidence (4\%). However this is not significant at $\mathrm{p}=0.05$ level of confidence. 
- The most common age group of the subjects exposed to intentional attempt and adding to the mortality is $15-24$ years (39\%) followed by age groups of $25-54$ years $(35 \%)$. Intentional intake $(91 \%)$ of the poison is the major type of attempt adding to mortality [p value $=0.008$ ]

- The most common agent used for intentional incidence and adding to the mortality is Organophosphates (67\%) followed by Phosphides (19\%). However this is not significant at $\mathrm{p}=0.05$ level of significance.

- Incidence of poisoning was seen high during rainy seasons $(24 \%)$ which is slightly higher than that of autumn (23\%). This however is not significant at $\mathrm{p}=$ 0.05 level of significance.

- The most frequent age group prone to intentional incidence is $15-24$ years (44\%) while children (below 15 years) were more frequent to accidental incidence of poisoning (6\%). This was statistically significant at $\mathrm{p}=0.000$

\section{CONCLUSIONS}

The study of poisoning cases in central, zonal, and district level hospitals and comparing and analyzing these cases have helped us to draw some important conclusions. The cases, as analyzed have presented us important results. It has clearly shown a strong relationship between the social parameter and the incidence of poisoning. There is also a relationship within the parameters.

Along with these social parameters, the availability of these agents has also added to the incidence. This is where we need to open our eyes. The implementation of the regulations so made needs to be "really implemented". The results also clearly show that drugs also have a significant role to play in these incidences. These drugs are mostly sedatives and hypnotics. Needless to say, the wide availability of these agents is the cause. Isn't this the prime time to look where we have deceived our profession?

We still need better management to be followed to reduce the mortality. New technologies can easily solve the cases that seem so complex to us. The government should know that the health care system of our country needs their major attention.

Our focus was drawn by the pesticide-Aluminium phosphide. This pesticide has a mortality of more than $50 \%{ }^{9}$. Even though the data which were collected from the six hospitals $(12 \%)$ had not supported this mortality rate but the data collected from NPIC (63\%) significantly supports the ill effects of this "devil in disguise". It clearly shows the increasing craze for this agent. However one should understand that our aim was only to study Aluminium Phosphide poisoning and not to give any treatment protocol or any decision regarding this pesticide. The data from NPIC and study of some more research work were reviewed. The study clearly showed that toxicologist has not yet been able to conquer Aluminium phosphide poisoning. However parenteral magnesium sulfate is a ray of hope.

\section{ACKNOWLEDGEMENT}

We would like to extend our gratitude to The Director of Nepal Poison Information Center for providing us the necessary information for the project. We would also like to 
thank the administration of Bir Hospital, Tribhuwan University Teaching hospital, Janakpur Zonal Hospital, Gandaki Zonal Hospital, Bhaktapur Hospital and Siraha District Hospital for providing us with the necessary information and data.

Our heartily thanks also goes to all our friends and staff members for all sorts of help and encouragement.

\section{REFERENCES}

1. Bekry A R A 2000. Trends in Sucide, Parasucide and Accidental Poisoning in Children in Adis Ababa, Ethiopia, Dept. of Psychiatry, Addis Ababa Medical University, Original Article.

2. Bharati U., Shrestha J. B. and Sharma M. 2001. Study of Acute Poisoning in Nepal Medical College Teaching Hospital; NMCJ Vol. 2 No. 2 (83).

3. Bruns J. J. (2000). Anticholinergic Toxicity; Original Article on www.emedicine.com.

4. Hobun T and Nahachi C (1996) A Study of Organophosphate Poisoning Cases at Parirenyatwa Hospital,Zimwabe, www. uz.ac.zw/medicine/pharmacy.

5. http/members.tripoid,com/Prof_Anil_Aggrawal/Poiso007.htm, www.Phosphine.com.

6. Kafle K.K. 1989. Poisoning cases at T.U Teaching Hospital, J. Inst. Med., 11, 297-301.

7. Kafle K.K., Gyawali K.K., 1992. Organophosphorous Most common poisoning Agent Original Article-6, J. Inst., Med.,14,228-223.

8. Lohani S. P. 2000. An epidemiological study on acute pyrethroid poisoning; original article.

9. Lohani S P, Joshi B D., Banskota H K. 1998. Hydrocarbon Ingestion: Case Report and Review of Literature; NMCJ Vol 2 No.1 (5).

10. Lohani S. P. 1995. Deliberate self-poisoning in the SAARC Countries, original article.

11. Pokhrel Nita , Gurung C K 1987. A study of poisoning case recorded in Bir hospital over four years, J. Inst. Med., 29-24.

12. Sobhani A R, 1995). Drug and Poisoning in Northern Iran, original article.

13. Suman R.L. and Savani Minal 1999. Pleural effusion_A Rare Complication of Aluminium Phosphide Poisoning; Indian Pediatrics;36: 1161-1163;1 From the Department of Pediatrics, Bal Chikitsalya, R.N.T. Medical College, Udaipur, Rajasthan, India. www.indianpediatrics.net/links.htm. 
KATHMANDU UNIVERSITY JOURNAL OF SCIENCE, ENGINEERING AND TECHNOLOGY

VOL. I, No. V, SEPTEMBER 2008, pp 40-48.

14. Vaidya H R, Shrestha Yogesh 1999. Changing trends of Adult Poisoning cases at Patan Hospital, Original article published in

15. www.doctorsaab.com, Dangers to First-aiders

16. www.mednets.com/toxicology.htm, Phosphine Fumigants. 